PROCEEDINGS OF THE

AMERICAN MATHEMATICAL SOCIETY

Volume 132, Number 10, Pages 3089-3095

S 0002-9939(04)07308-3

Article electronically published on May 20, 2004

\title{
SUMMABLE PROCESSES VERSUS SEMIMARTINGALES
}

\author{
NICOLAE DINCULEANU AND OANA MOCIOALCA
}

(Communicated by Richard C. Bradley)

\begin{abstract}
The classical stochastic integral $\int H d X$ is defined for real-valued semimartingales $X$. For processes with values in a Banach space $E$, the stochastic integral $\int H d X$ is defined for locally summable processes $X$, using a measure-theoretical approach.

We investigate the relationship between semimartingales and locally summable processes.

A real-valued, locally summable process is a special semimartingale. We prove that in infinite-dimensional Banach spaces, a locally summable process is not necessarily a semimartingale.
\end{abstract}

\section{INTRODUCTION}

The purpose of this paper is to prove that there are locally summable processes (in the sense of Definition 5 below) with values in an infinite-dimensional Banach space $E$ that are not semimartingales. An $E$-valued semimartingale is defined to be the sum of a local martingale and a process with finite variation. (All local martingales are assumed to be cadlag; all processes with finite variation or finite semivariation are assumed to be cadlag and adapted.)

For real-valued processes, Kussmaul proved in [K], Theorem 12.3, that a locally summable process (in the sense of Definition 5 below) is a semimartingale, i.e. the sum of a local martingale and a predictable process of finite variation. (We remark that Kussmaul uses a different definition of local summability, which is called prelocal summability in [D-M], VII.97.) But a semimartingale is not necessarily locally summable. For example, an increasing process is not (locally) summable, unless it is (locally) integrable.

For processes with values in a Banach space $E$, a process with integrable variation is summable relative to $(\mathbb{R}, E)([\mathrm{D}$, Theorem 19.13); and a process with integrable semivariation relative to ( $\mathbb{R}, E$ ) is summable relative to $(\mathbb{R}, E)$, provided that $c_{0} \notin E$ ([D], Theorem 21.12). A (locally) square integrable (local) martingale is (locally) summable provided $E$ is a Hilbert space ([D], Theorem 17.7 and Corollary 17.7); but if $E$ is not a Hilbert space, a square integrable martingale might not be summable.

In this paper we prove that if $E$ is infinite dimensional, the converse implication is not true either: a locally summable process is not necessarily a semimartingale (Theorem 8). This follows from the main theorem of the paper, which states

Received by the editors August 27, 2002.

2000 Mathematics Subject Classification. Primary 60H05; Secondary 60G20.

Key words and phrases. Summable processes, semimartingale, integrable variation, integrable semivariation, local martingale, dual projection. 
that if $c_{0} \not \subset E$ and $E$ has the Radon-Nikodým Property (RNP), a predictable semimartingale with finite semivariation relative to $(\mathbb{R}, E)$ has necessarily finite variation (Theorem 6). This is a general theorem on stochastic processes and does not involve the summability or the stochastic integral. The conditions $c_{0} \not \subset E$ and $E \in(\mathrm{RNP})$ are not too restrictive; they are satisfied by any reflexive Banach space, in particular by a Hilbert space.

In Theorem 8 we give a very simple example of a deterministic, summable process that has integrable semivariation and infinite variation; therefore, it is not a semimartingale.

Remark. The process in this example was considered in [B-D] in the extreme particular case where $\Omega$ consists of a single point. In this case any $E$-valued process is a function $f: \mathbb{R}_{+} \rightarrow E$, and any martingale is constant, hence with finite variation. Therefore any semimartingale has finite variation. It follows that, in this case, the above example is not a semimartingale. A direct computation shows that the above process is summable relative to $(\mathbb{R}, E)$ without imposing any conditions on $E$.

However, if $\Omega$ is arbitrary, a semimartingale might have infinite variation. The process in the above example also has infinite variation, and the proof of Theorem 6 that ensures that it is not a semimartingale is quite complicated, involving a whole series of results such as the existence of predictable dual projections for $E$-valued measures and for processes with integrable variation, the Lebesgue-Stieltjes integral with respect to functions with finite semivariation (rather than finite variation) and other results for vector-valued stochastic processes that can be found in [D].

\section{Preliminaries}

In this paragraph, we present the notation that will be used throughout the paper and state the definitions of summability and of semivariation, for measures, functions and processes. For all the notions and results used in the sequel, we refer the reader to $[\mathrm{D}-\mathrm{M}]$ and $[\mathrm{D}]$.

2.1. Notation. $(\Omega, \mathcal{F}, P)$ is a probability space; $\left(\mathcal{F}_{t}\right)_{t \in \mathbb{R}_{+}}$is a filtration satisfying the usual conditions; $\mathcal{R}$ is the ring generated by the semiring of predictable rectangles of the form $\{0\} \times A$ with $A \in \mathcal{F}_{0}$ and $(s, t] \times A$ with $0 \leq s \leq t<\infty$ and $A \in \mathcal{F}_{s} ; \mathcal{P}$ is the predictable $\sigma$-algebra generated by the ring $\mathcal{R}$.

We denote $\mathcal{M}=\mathcal{B}\left(\mathbb{R}_{+}\right) \times \mathcal{F}$. Processes measurable with respect to $\mathcal{M}$ will be called, simply, measurable processes.

$E, F, G$ are Banach spaces with $E \subset L(F, G)$; for example, $E=L(\mathbb{R}, E), E \subset$ $L\left(E^{*}, \mathbb{R}\right), E=L(E, \mathbb{R})$ if $E$ is a Hilbert space.

For any Banach space $M$ we denote by $|\cdot|$ its norm, by $M^{*}$ its dual and by $M_{1}$ its unit ball.

2.2. Semivariation of measures. Let $S$ be a nonempty set, $\mathcal{S}$ a ring of subsets of $S$ and $m: \mathcal{S} \rightarrow E \subset L(F, G)$ an additive measure.

Definition 1. The semivariation of $m$ on a set $A \subset S$ relative to the embedding $E \subset L(F, G)$, or relative to the pair $(F, G)$, is a number $\tilde{m}_{F, G}(A)$ defined by

$$
\tilde{m}_{F, G}(A)=\sup \left|\sum_{i \in I} m\left(A_{i}\right) x_{i}\right|
$$

where the supremum is taken for all finite families $\left(A_{i}\right)_{i \in I}$ of disjoint sets from $\mathcal{S}$ contained in $A$ and all families $\left(x_{i}\right)_{i \in I}$ of elements from $F$ with $\left|x_{i}\right| \leq 1$. 
We say that $m$ has finite (respectively bounded) semivariation relative to $(F, G)$ if $\tilde{m}_{F, G}(A)<\infty$ for every $A \in \mathcal{S}$ (respectively $\tilde{m}_{F, G}(S)<\infty$ ).

For a detailed account of integration with respect to $\sigma$-additive measures with finite semivariation, see $[\mathrm{D}], \S 5$.

2.3. Semivariation of functions and their Lebesgue-Stieltjes integral. Let $g: I \rightarrow E \subset L(F, G)$ be a function.

Definition 2. The semivariation of $g$ on any interval $I \subset \mathbb{R}$, relative to $(F, G)$ is the number $\operatorname{svar}_{F, G}(g, I)$ defined by

$$
\operatorname{svar}_{F, G}(g, I)=\sup \left|\sum_{i=1}^{n}\left[g\left(t_{i+1}\right)-g\left(t_{i}\right)\right] x_{i}\right|,
$$

where the supremum is taken for all finite divisions $t_{1}<t_{2}<\cdots<t_{n+1}$ consisting of points of $I$ and all finite families $\left(x_{1}, x_{2}, \ldots, x_{n}\right)$ of elements of $F$ with $\left|x_{i}\right| \leq 1$.

We denote $\tilde{g}_{F, G}(t)=\operatorname{svar}_{F, G}(g,(-\infty, t])$, for $t \in \mathbb{R}$.

We say that $g$ has finite (resp. bounded) semivariation relative to $(F, G)$ if $\operatorname{svar}_{F, G}(g, I)<\infty$ for every bounded interval $I\left(\operatorname{resp} \operatorname{svar}_{F, G}(g, \mathbb{R})<\infty\right)$.

To the function $g$ we associate a measure $m_{g}: \mathcal{S} \rightarrow E \subset L(F, G)$ on the ring $\mathcal{S}$ generated by the intervals $(a, b]$, defined by

$$
m_{g}(a, b]=g(b)-g(a),
$$

and extended by additivity to $\mathcal{S}$.

The measure $m_{g}$ has finite (resp. bounded) semivariation relative to $(F, G)$ iff $g$ has finite (resp. bounded) semivariation relative to $(F, G)$.

Theorem 3 ([D] , Theorem 20.13). Assume $c_{0} \not \subset E$ and that $g$ is right continuous and has finite (resp. bounded) semivariation relative to $(\mathbb{R}, E)$. Then $m_{g}$ can be extended uniquely to a $\sigma$-additive measure $m: \mathcal{D} \rightarrow E($ resp. $m: \mathcal{B}(\mathbb{R}) \rightarrow E$ ) with finite (resp. bounded) semivariation relative to $(\mathbb{R}, E)$, where $\mathcal{D}$ is the $\delta$-ring of all bounded Borel subsets of $\mathbb{R}$. If $g$ has finite (resp. bounded) semivariation relative to $(\mathbb{R}, E)$ and relative to $(F, G)$, then the extension $m$ has finite (resp. bounded) semivariation relative to $(F, G)$.

If $f: S \rightarrow F$ is a function and the integral $\int f d m$ is defined, we denote it by $\int f d g$ and we call it the Lebesgue-Stieltjes integral of $f$ with respect to $g$. For more information about functions with finite semivariation and their Stieltjes integral, see [D], $\S 20$.

2.4. Processes with finite semivariation. Let $X: \mathbb{R}_{+} \times \Omega \rightarrow E \subset L(F, G)$ be a process. We consider the processes extended to $\mathbb{R} \times \Omega$ with $X_{t}(\omega)=0$ for $t<0$.

Definition 4. We say that $X$ has finite (resp. bounded) semivariation relative to $(F, G)$, if for each $\omega \in \Omega$, the path $X$. $(\omega)$ has finite (resp. bounded) semivariation relative to $(F, G)$.

For each $t \geq 0$ we denote $\tilde{X}_{F, G}(t, \omega)=\operatorname{svar}_{F, G}(X .(\omega),(-\infty, t])$. If the pair $(F, G)$ is understood, we write $\tilde{X}$ instead of $\tilde{X}_{F, G}$ and $\tilde{X}_{t}(\omega)=\tilde{X}_{F, G}(t, \omega)$.

We say that $X$ has locally integrable (resp. integrable) semivariation relative to $(F, G)$ if $E\left(\tilde{X}_{t}\right)<\infty$ for every $t \in \mathbb{R}_{+}$(resp. $\left.E\left(\tilde{X}_{\infty}\right)<\infty\right)$.

For a detailed account of processes with finite semivariation, see [D], $\S 21$. 
2.5. Summable processes. Let $X: \mathbb{R}_{+} \times \Omega \rightarrow E$ be a cadlag, adapted process, with $X_{t} \in L_{E}^{1}$ for every $t \geq 0$. We define the additive measure $I_{X}: \mathcal{R} \rightarrow L_{E}^{1} \subset$ $L\left(F, L_{G}^{1}\right)$, first for predictable rectangles, by $I_{X}(\{0\} \times A)=1_{A} X_{0}$ for $A \in \mathcal{F}_{0}$, and $I_{X}((s, t] \times A)=1_{A}\left(X_{t}-X_{s}\right)$ for $A \in \mathcal{F}_{s}$, and then, extended by additivity to $\mathcal{R}$.

Definition 5. We say that $X$ is summable relative to the embedding $E \subset L(F, G)$ or relative to the pair $(F, G)$ if $I_{X}$ has a $\sigma$-additive extension $I_{X}: \mathcal{P} \rightarrow L_{E}^{1} \subset L\left(F, L_{G}^{1}\right)$ with finite semivariation relative to the pair $\left(F, L_{G}^{1}\right)$. We say that $X$ is locally summable relative to $(F, G)$ if there is an increasing sequence $\left(T_{n}\right)$ of stopping times with $T_{n} \uparrow \infty$, such that, for each $n$, the stopped process $X^{T_{n}}$ is summable relative to $(F, G)$.

If $X$ is a locally summable process, one can define the stochastic integral, denoted by $H \circ X$ or $\int H d X$, for certain predictable, $F$-valued processes $H$. For the construction of the stochastic integral the reader is referred to $[\mathrm{D}$, chapter 2.

For the definition of the classical stochastic integral $\int H d X$ with respect to realvalued semimartingales, the reader is referred to $[\mathrm{D}-\mathrm{M}]$, chapter viii.

In this paper we shall not be concerned with the stochastic integral, but only with the summability.

\section{Summability Versus SEMimartingales}

The definition of $E$-valued local martingales and semimartingales is the same as for real-valued processes (see [D-M], Definitions vii.27 and vii.23). In Theorem 6 we prove that a right continuous, predictable semimartingale with finite semivariation has necessarily locally integrable variation. Then in Theorem 8 we give a simple example of a deterministic, right continuous, predictable process with integrable semivariation and summable, but with infinite variation (and therefore not a semimartingale).

Theorem 6. Let $E$ be a Banach space with $c_{0} \not \subset E$, having the Radon-Nikodým Property (RNP). Let $X: \mathbb{R}_{+} \times \Omega \rightarrow E$ be a right continuous, predictable semimartingale with finite semivariation $\tilde{X}_{\mathbb{R}, E}$. Then $X$ has locally integrable semivariation $\tilde{X}_{\mathbb{R}, E}$ and locally integrable variation $|X|$.

Proof. To simplify the writing, the semivariation relative to $(\mathbb{R}, E)$ of any process $Y$ will be denoted by $\widetilde{Y}$ instead of $\widetilde{Y}_{\mathbb{R}, E}$.

a) Since $X$ is predictable, it has separable range; therefore, we can assume that $E$ is separable.

Since $X$ is a right continuous semimartingale, we have $X=M+A$, where $M$ is a right continuous local martingale and $A$ is a right continuous, adapted process with finite variation $|A|$. Then $A$ has finite semivariation $\tilde{A}$. From $M=X-A$ we deduce that $M$ has finite semivariation $\tilde{M}$. Since $X$ is a right continuous, predictable process with finite semivariation $\tilde{X}$ and $E$ is separable, by Theorem 22.15 in D we deduce that $X$ has locally integrable semivariation $\tilde{X}$. Since $c_{0} \not \subset E$ and $M$ is a right continuous local martingale with finite semivariation $\tilde{M}$, by Theorem 22.16 in [D], $M$ has locally integrable semivariation $\tilde{M}$. It follows that $A$ has locally integrable semivariation $\tilde{A}$.

b) $A$ has locally integrable variation $|A|$. In fact, there is an increasing sequence $\left(S_{n}\right)$ of stopping times with $S_{n} \uparrow \infty$ such that we have $E\left(\tilde{A}_{S_{n}}\right)<\infty$ for every $n$. 
For each $n$ we define the stopping time $T_{n}$ by

$$
T_{n}=S_{n} \wedge \inf \left\{t:|A|_{t} \geq n\right\} .
$$

It follows that $|X|_{T_{n}-} \leq n$. Since $A$ has finite variation, by Theorem 18.20 in $\mathrm{D}$, the jumps of $A_{T_{n}}$ and $\left|A_{T_{n}}\right|$ satisfy

$$
\Delta|A|_{T_{n}}=\left|\Delta A_{T_{n}}\right| .
$$

Moreover, from the inequality

$$
\left|A_{t}-A_{s}\right| \leq \operatorname{svar}(A,(-\infty, t])=\tilde{A}_{t}, \text { for } s \leq t,
$$

taking left limits as $s \uparrow t$ we get $\left|\Delta A_{t}\right| \leq \tilde{A}_{t}$. In particular, $\left|\Delta A_{T_{n}}\right| \leq \tilde{A}_{T_{n}}$; hence $\Delta|A|_{T_{n}} \leq \tilde{A}_{T_{n}}$.

From $\Delta|A|_{T_{n}}=|A|_{T_{n}}-|A|_{T_{n}-}$ we deduce that $|A|_{T_{n}}=|A|_{T_{n}-}+\Delta \mid A_{T_{n}} \leq$ $n+\tilde{A}_{T_{n}}$. Therefore $E\left(|A|_{T_{n}}\right) \leq n+E\left(\tilde{A}_{T_{n}}\right)<\infty$; hence $A$ has locally integrable variation $|A|$.

c) We assume first that $X$ has integrable semivariation $\tilde{X}$ and that $X=M+A$, where $M$ is a right continuous, uniformly integrable martingale with integrable semivariation $\tilde{M}$ and $A$ is a right continuous, adapted process with integrable variation $|A|$.

Let $z \in E^{*}$. Then $\langle X, z\rangle=\langle M, z\rangle+\langle A, z\rangle$. The processes $\langle X, z\rangle,\langle M, z\rangle$, and $\langle A, z\rangle$ are adapted, right continuous and have integrable variation; $\langle X, z\rangle$ is predictable and $\langle M, z\rangle$ is a uniformly integrable martingale. Consider the $\sigma$-additive stochastic measures $\mu_{\langle X, z\rangle}, \mu_{\langle M, z\rangle}, \mu_{\langle A, z\rangle}: \mathcal{M} \rightarrow \mathbb{R}$ with finite variation, defined for any real-valued, bounded, measurable process $\phi$ by $\int \phi d \mu_{\langle X, z\rangle}=E\left(\int \phi d\langle X, z\rangle\right)$, $\int \phi d \mu_{\langle M, z\rangle}=E\left(\int \phi d\langle M, z\rangle\right)$, and $\int \phi d \mu_{\langle A, z\rangle}=E\left(\int \phi d\langle A, z\rangle\right)$ (see [D-M], vi.64). Then $\mu_{\langle X, z\rangle}=\mu_{\langle M, z\rangle}+\mu_{\langle A, z\rangle}$. Since $\langle M, z\rangle$ is a uniformly integrable martingale with integrable variation, we have $\mu_{\langle M, z\rangle}(B)=0$ for $B \in \mathcal{P}$. It follows that $\mu_{\langle X, z\rangle}=$ $\mu_{\langle A, z\rangle}$ on $\mathcal{P}$.

Let $\phi$ be a real-valued, bounded, measurable process, and let ${ }^{p} \phi$ be its predictable projection. Then

$$
\int{ }^{p} \phi d \mu_{\langle X, z\rangle}=\int{ }^{p} \phi d \mu_{\langle A, z\rangle} \text {, i.e., } E\left(\int{ }^{p} \phi d\langle X, z\rangle\right)=E\left(\int{ }^{p} \phi d\langle A, z\rangle\right) .
$$

Since $\langle X, z\rangle$ is predictable, we have $E\left(\int{ }^{p} \phi d\langle X, z\rangle\right)=E\left(\int \phi d\langle X, z\rangle\right)$. It follows that

$$
E\left(\int \phi d\langle X, z\rangle\right)=E\left(\int{ }^{p} \phi d\langle A, z\rangle\right), \text { or }\left\langle E\left(\int \phi d X\right), z\right\rangle=\left\langle E\left(\int{ }^{p} \phi d A\right), z\right\rangle,
$$

where, for each $\omega \in \Omega$, the integrals are Stieltjes integrals with respect to the functions $X(\omega)$ and $A(\omega)$ with finite semivariation (see 20.15 in [D]). Since $z \in E^{*}$ was arbitrary, we deduce that

$$
E\left(\int \phi d X\right)=E\left(\int{ }^{p} \phi d A\right) .
$$

It follows that $X$ is the predictable dual projection with integrable semivariation $\tilde{X}$ of the process $A$ with integrable semivariation $\tilde{A}$ (see Definition 22.4 in [D]).

By Theorem 19.8 in [D], there is a $\sigma$-additive stochastic measure $\mu_{A}: \mathcal{M} \rightarrow E$ with finite variation $\left|\mu_{A}\right|$ satisfying $\int \phi d \mu_{A}=E\left(\int \phi_{s} d A_{s}\right)$, for any real-valued, bounded, measurable process $\phi$. Consider the predictable dual projection $\mu_{A}^{p}$ of $\mu_{A}$ (see Definition 22.2 in [D]). By Theorem 22.1 in [D], $\mu_{A}^{p}$ has finite variation $\left|\mu_{A}^{p}\right|$. By Proposition 22.3 in [D], we have $\int \phi d \mu_{A}^{p}=\int{ }^{p} \phi d \mu_{A}$, for any real-valued, 
measurable, bounded process $\phi$. Since $E$ has the RNP, we can apply Theorem $19.12 \mathrm{~d})$ in [D] for the measure $\mu_{A}^{p}: \mathcal{M} \rightarrow E=L(\mathbb{R}, E)$ and deduce the existence of a right continuous predictable process $Y: \mathbb{R}_{+} \times \Omega \rightarrow L(\mathbb{R}, E)$, with integrable variation $|Y|$, such that, for any real-valued, measurable, bounded process $\phi$ we have $\int \phi d \mu_{A}^{p}=E\left(\int \phi_{s} d Y_{s}\right)$; hence

$$
E\left(\int \phi_{s} d Y_{s}\right)=E\left(\int{ }^{p} \phi_{s} d A_{s}\right)
$$

where the integrals are Stieltjes integrals with respect to the processes with finite variation (see [D] 18.21) which, by Remark 20.37 in $[\mathrm{D}$, are equal to the Stieltjes integrals with respect to the same processes, considered with finite semivariation. It follows that $E\left(\int \phi_{s} d Y_{s}\right)=E\left(\int \phi_{s} d X_{s}\right)$ for every real-valued measurable, bounded process $\phi$.

If $T$ is any stopping time, we take $\phi=1_{(T, \infty)}$ and obtain $E\left(Y_{\infty}-Y_{T}\right)=$ $E\left(X_{\infty}-X_{T}\right)$. Since both $X$ and $Y$ are predictable and $c_{0} \notin E$, by Corollary $\left.21.10 \mathrm{~b}^{\prime}\right)$ in $\mathrm{D}$, $X$ and $Y$ are indistinguishable. It follows that $X$ has integrable variation $|X|$.

d) We now prove the theorem in the general case, assuming that $X=M+A$ where $M$ is a right continuous, local martingale with $M_{0}=0$ and $A$ is a right continuous, adapted process with finite variation. By step a), the processes $X$ and $M$ have locally integrable semivariation relative to $(\mathbb{R}, E)$. By step b), $A$ has locally integrable variation $|A|$.

Let $\left(T_{n}\right)$ be an increasing sequence of stopping times with $T_{n} \uparrow \infty$ such that for each $n, X^{T_{n}}$ and $M^{T_{n}}$ have integrable semivariation relative to $(\mathbb{R}, E), M^{T_{n}}$ is a uniformly integrable martingale and $A^{T_{n}}$ has integrable variation. For each $n$ we have $X^{T_{n}}=M^{T_{n}}+A^{T_{n}}$. By step c), $X^{T_{n}}$ has integrable variation for each $n$. It follows that $X$ has locally integrable variation.

The following corollary is an immediate useful consequence of Theorem 6 .

Corollary 7. Let $E$ be a Banach space with $c_{0} \notin E$, having the RNP. Let $X$ : $\mathbb{R}_{+} \times \Omega \rightarrow E$ be a right continuous, predictable process with finite semivariation $\tilde{X}_{\mathbb{R}, E}$. Assume that the set of points $\omega \in \Omega$ such that $X .(\omega)$ has infinite variation is not negligible. Then $X$ is not a semimartingale.

Theorem 8. Let $E$ be an infinite-dimensional Banach space with $c_{0} \not \subset E$, having the $R N P$. There are E-valued summable processes relative to $(\mathbb{R}, E)$ that are not semimartingales.

Proof. We shall construct a simple example of an $E$-valued, right continuous predictable, deterministic process with integrable semivariation relative to $(\mathbb{R}, E)$, having infinite variation. By Theorem 21.12 in [D] such a process is summable relative to $(\mathbb{R}, E)$. By Corollary 8 , such a process cannot be a semimartingale. The construction of the example does not require the condition $c_{0} \not \subset E$ or RNP.

Using the Dvoretzky-Rogers theorem, we can find a sequence $\left(x_{n}\right)_{1 \leq n \leq \infty}$ of elements of $E$, such that the series $\sum_{n=1}^{\infty} x_{n}$ is unconditionally convergent but not absolutely convergent.

Denote $s_{0}=0, s_{1}=x_{1}, \cdots, s_{n}=\sum_{i=1}^{i=n} x_{i}, \cdots$. Then $x_{n}=s_{n}-s_{n-1}, \lim _{n \rightarrow \infty} s_{n}$ $=\sum_{i=1}^{\infty} x_{i}$ and $\sum_{n=1}^{\infty}\left|s_{n}-s_{n-1}\right|=\infty$.

Consider the function $g: \mathbb{R}_{+} \rightarrow E$ defined by $g(0)=\sum_{n=1}^{\infty} x_{n}$ and $g(t)=$ $\sum_{1 \leq n<\infty} 1_{\left[\frac{1}{n+1}, \frac{1}{n}\right)}(t) s_{n}$, for $t>0$. We define the deterministic process $X: \mathbb{R}_{+} \times$ 
$\Omega \rightarrow E$ by $X=g 1_{\Omega}$, i.e., $X_{t}(\omega)=g(t)$ for $t \geq 0$ and $\omega \in \Omega$. We shall prove that $X$ is a right continuous predictable process with finite, integrable semivariation $\tilde{X}_{\mathbb{R}, E}$ and with infinite variation.

The proof is divided into several steps.

a) From the definition of $g$ we deduce that $g$ is right continuous; therefore, $X$ is right continuous.

b) $g$ has infinite variation on $\mathbb{R}_{+}$; hence $X$ has infinite variation on $\mathbb{R}_{+}$. In fact, we have $g(t)=s_{n}$ if $\frac{1}{n+1} \leq t<\frac{1}{n}$. We have then for each $k$,

$$
\sum_{n=1}^{k}\left|g\left(\frac{1}{n}\right)-g\left(\frac{1}{n+1}\right)\right|=\sum_{n=1}^{k}\left|s_{n-1}-s_{n}\right|=\sum_{n=1}^{k}\left|x_{n}\right| .
$$

It follows that $\operatorname{var}\left(g, \mathbb{R}_{+}\right) \geq \sup _{k} \sum_{n=1}^{k}\left|g\left(\frac{1}{n}\right)-g\left(\frac{1}{n+1}\right)\right|=\sup _{k} \sum_{n=1}^{k}\left|x_{n}\right|=$ $\sum_{n=1}^{\infty}\left|x_{n}\right|=\infty$.

c) $g$ has bounded semivariation $\tilde{g}=\tilde{g}_{\mathbb{R}, E}$ on $\mathbb{R}_{+}$; in fact, let $x^{*} \in E^{*}$. The jumps of $x^{*} g$ are at 0 , equal to $x^{*} g(0)$ and at $\frac{1}{n}$, equal to $x^{*} s_{n-1}-x^{*} s_{n}$. Therefore,

$$
\begin{aligned}
\operatorname{svar}_{\mathbb{R}, E}\left(x^{*} g, \mathbb{R}_{+}\right) & =\left|x^{*} g(0)\right|+\sum_{n=1}^{\infty}\left|x^{*} s_{n-1}-x^{*} s_{n}\right| \\
& \leq \sum_{n=1}^{\infty}\left|x^{*}\left(x_{n}\right)\right|+\sum_{n=1}^{\infty}\left|x^{*}\left(s_{n-1}-s_{n}\right)\right| \\
& =2 \sum_{n=1}^{\infty}\left|x^{*}\left(x_{n}\right)\right|<\infty .
\end{aligned}
$$

Then, by Proposition 20.9 in [D] we have $\operatorname{svar}_{\mathbb{R}, E}\left(g, \mathbb{R}_{+}\right)<\infty$; hence $g$ has bounded semivariation $\tilde{g}$. From $\tilde{X}_{t}(\omega)=\tilde{g}(t)$ we deduce that $X$ has bounded semivariation $\tilde{X}$.

d) $X$ has integrable semivariation $\tilde{X}=\tilde{X}_{\mathbb{R}, E}$. In fact,

$$
E\left(\tilde{X}_{\infty}\right)=\int \tilde{X}_{\infty} d P=\int \operatorname{svar}_{\mathbb{R}, E}\left(X, \mathbb{R}_{+}\right) d P=\operatorname{svar}_{\mathbb{R}, E}\left(g, \mathbb{R}_{+}\right)<\infty
$$

e) $X$ is adapted since it is deterministic.

f) $X$ is predictable since the sets $\{0\} \times \Omega$ and $\left[\frac{1}{n+1}, \frac{1}{n}\right) \times \Omega$ are predictable.

\section{REFERENCES}

[B-D] J. K. Brooks and N. Dinculeanu, Stochastic Integration in Banach Spaces, Seminar on Stochastic Processes, Birkhäuser, Boston, 1991, 27-115.

[D-M] C. Dellacherie and P. A. Meyer, Probabilités et Potentiel, Hermann, Paris, 1975-1980. MR 58:7757

[D] N. Dinculeanu, Vector Integration and Stochastic Integration in Banach Spaces, Wiley, New York, 2000. MR 2001h:60096

[K] A. U. Kussmaul, Stochastic integration and Generalized Martingales, Pitman, London, 1977. MR 58:7841

Department of Mathematics, University of Florida, 358 Little Hall, P.O. Box 118105, Gainesville, Florida 32611-8105

E-mail address: nd@math.ufl.edu

Department of Mathematics, Purdue University, 150 N. University Street, West LAFAYETTE, Indiana 47907-2067

E-mail address: oana@math.purdue.edu 